\title{
Gastric and Endobronchial Metastases in a Case of Lobular Breast Cancer
}

\author{
G.S. Fernandes ${ }^{a} \quad$ T.S. Corrêa ${ }^{b} \quad$ E.P.B. Carvalho ${ }^{a} \quad$ A. Katz ${ }^{a} \quad$ P.M. Hoff $f^{a b}$ \\ ${ }^{a}$ Oncology Center, Hospital Sírio-Libanês, Unidade Brasília, Brasília, and ${ }^{\text {b} I n s t i t u t o ~ d o ~}$ \\ Câncer do Estado de São Paulo, São Paulo, Brazil
}

\section{Key Words}

Lobular breast cancer · Gastric metastasis · Endobronchial metastasis · Invasive lobular carcinoma

\begin{abstract}
Background: Invasive lobular carcinoma (ILC) is the second most common histological type of invasive breast carcinoma, preceded only by infiltrating ductal carcinoma, which has clinical, biological and molecular distinctions. These distinctions imply a different metastatic behavior between the histology of these 2 types of breast cancer. Case Presentation: We report the case of a 51-year-old woman with breast cancer with ILC histology, diagnosed at an early stage. In the course of her disease, recurrences in the gastric mucosa and endobronchial area occurred. The treatment she received is described herein. Conclusion: This is a case of ILC with unusual metastases. The absence of $\mathrm{E}$-cadherin is related to the carcinogenesis of ILC and probably to these patterns of metastasis as well.
\end{abstract}

(c) 2013 S. Karger AG, Basel

\section{Introduction}

Invasive lobular carcinoma (ILC) is the second most common histological type of invasive breast carcinoma, preceded only by infiltrating ductal carcinoma, which has clinical, biological and molecular distinctions. ILC tends to be larger in size at diagnosis and most often affects older women. It usually occurs bilaterally, involving multicentric tumors, and there is greater difficulty in detecting lesions in mammograms. In addition, most ILC displays estrogen (ER) receptor expression and low histological grade. There is also a different

G.S. Fernandes

Centro de Oncologia, Hospital Sírio-Libanês, Unidade Brasília

SGAS 613, Asa Sul

Brasília, DF $70200-730$ (Brazil)

E-Mail gustavo.hemato@gmail.com 
Fernandes et al.: Gastric and Endobronchial Metastases in a Case of Lobular Breast Cancer

pattern of metastatic dissemination, and later involvement of unusual sites such as the peritoneum, meninges and gastrointestinal tract [1-3].

\section{Case Presentation}

A 51-year-old female patient underwent quadrantectomy with right axillary dissection on May 31, 1999. Pathological examination showed a 1-cm ILC, histological grade I, and no lymph node involvement. The immunohistochemical analysis showed high expression of ER $(100 \%)$ and progesterone (PR, 100\%) receptors.

The adjuvant chemotherapy AC regimen [Adriamycin $60 \mathrm{mg} / \mathrm{m}^{2}$ and cyclophosphamide $600 \mathrm{mg} / \mathrm{m}^{2}$, both on day (D) 1, every 21 days] was given for 4 cycles, followed by adjuvant radiotherapy and hormone therapy with tamoxifen $20 \mathrm{mg}$ /day for 5 years until March 2005 .

In May 2005, due to a recent onset of dyspeptic symptoms, the patient underwent an upper endoscopy, which showed an infiltrative lesion involving the bottom half of the proximal gastric body. The biopsy revealed adenocarcinoma, but an immunohistochemical analysis was not performed. The staging examinations showed no evidence of metastatic disease, and based on the hypothesis of a primary neoplasm of the stomach, the patient underwent a total gastrectomy. The immunohistochemical analysis performed later on the gastrectomy specimen showed ER and PR expression and a lack of HER2 expression, favoring the diagnosis of breast cancer recurrence in the gastric mucosa.

After resection, based on the hypothesis of a second primary, there was no evidence of disease, and treatment was continued with adjuvant chemotherapy with 8 cycles of docetaxel $75 \mathrm{mg} / \mathrm{m}^{2}$. Afterward, the patient received hormone therapy with anastrozole, maintained until March 2009, when disease progression was detected in the bones. A new biopsy showed persistence of the previous immunohistochemical findings.

At that time, bone pain and dyspnea were noted upon moderate physical exercise. Chest tomography did not show lung parenchyma involvement, but there was a tapering distal to the right main bronchus suggestive of secondary involvement of the bronchial mucosa, which was confirmed by pathological examination (fig. 1).

The patient underwent brachytherapy with a high dose of radiation (fig. 2, fig. 3) for bronchial lesion treatment. In April 2009, first-line palliative chemotherapy with paclitaxel $80 \mathrm{mg} / \mathrm{m}^{2}$ on D1, D8 and D15, every 28 days, was started, in combination with Avastin 10 $\mathrm{mg} / \mathrm{kg}$ on D1 and D15. Treatment was discontinued only in July 2010 due to bone disease progression detected by PET-CT.

From July 2010 to November 2010, the patient received second-line hormone therapy with Faslodex, presenting symptomatic progression of bone disease after 5 months of treatment. In October 2010, third-line chemotherapy with capecitabine $1,750 \mathrm{mg} / \mathrm{m}^{2}$ from D1 to D14, every 21 days, was started. The patient maintained stable disease until October 2011, when she developed disease progression and was treated with everolimus plus an aromatase inhibitor. In March 2012, the patient presented with bone disease progression and was treated with radiotherapy. She developed an ocular metastasis when radiotherapy followed by chemotherapy (vinorelbine plus gemcitabine) was indicated. After new bone disease progression in July 2012, treatment was changed to liposomal doxorubicin 35 $\mathrm{mg} / \mathrm{m}^{2}$ until October 2012, when a peritoneal metastasis was detected; carboplatin plus paclitaxel was initiated. The patient developed meningeal metastasis and died in January 2013. 
Fernandes et al.: Gastric and Endobronchial Metastases in a Case of Lobular Breast Cancer

\section{Discussion}

Although pulmonary and pleural metastases are common in patients with solid tumors, endobronchial involvement is often of minor importance. A prevalence of 1.5 to $25 \%$ has been reported [4,5], whereas it may occur in approximately $18-42 \%$ of cases in patients with pulmonary metastasis [6]. Among primary tumors, breast cancer is the main cause of endobronchial metastasis, followed by colorectal and kidney cancer [7]. The use of high-dose brachytherapy, administered intraluminally, is an excellent alternative for the rapid palliation of symptoms for these lesions because it is safe and can be combined with systemic treatment, as illustrated in this clinical case (fig. 2).

Gastric metastases are uncommon and usually associated with melanoma and breast cancer. The estimated incidence in patients with breast cancer is $2-18 \%$, and it commonly occurs with other synchronic sites of metastases (90-94\%). The differentiation between metastasis and primary gastric cancer can be difficult based only on clinical, endoscopic and radiological findings; therefore, pathological and immunohistochemical examinations are needed. Although positivity for ER and PR is suggestive of metastatic breast carcinoma, this immunohistochemical result has been reported in $12-32 \%$ of primary neoplasms of the stomach [8]. These figures, however, come from studies using first-generation antibodies against the ER receptor $\beta$, which is no longer used in current practice.

In a more recent study that used second-generation antibodies against ER receptor $\alpha$ (ER $\alpha$ ), no cases of gastric cancer which exhibited positivity for ER $\alpha$ were reported. Thus, the positivity of ER $\alpha$ expression and the lack of E-cadherin were considered reliable for the definition of metastatic disease of the breast [9].

E-cadherin is a glycoprotein that contributes to adhesion between epithelial cells and plays a role in suppressing invasion and allowing the emergence of metastases. It is also involved in various cellular processes such as cell growth, migration, polarity and tissue integrity. Its expression is diminished or absent in several types of carcinomas including gastric, esophageal and hepatocellular.

The absence of E-cadherin expression is often used by pathologists to confirm the diagnosis of ILC. Its inactivation occurs irreversibly in more than $85 \%$ of cases of ILC, in general due to the loss of one allele combined with the mutation or methylation of the other allele [10-12]. This process seems to be an important step in the carcinogenesis of lobular carcinomas and possibly correlates with the pattern of metastases seen in this neoplasm, as illustrated in the present case.

\section{Conclusion}

This is a case of ILC with unusual metastases. The absence of E-cadherin is related to the carcinogenesis of ILC and is probably also related to these patterns of metastases. Therapeutic strategies aimed at the upregulation of E-cadherin may be promising in this context and deserve investigation.

\section{Acknowledgements}

We thank the patient for allowing us to report her case and learn more based on her disease. 


\begin{tabular}{l|l}
\hline DOI: $10.1159 / 000356564$ & $\begin{array}{l}\text { C 2013 S. Karger AG, Basel } \\
\text { www.karger.com/cro }\end{array}$ \\
\hline
\end{tabular}

Fernandes et al.: Gastric and Endobronchial Metastases in a Case of Lobular Breast Cancer

\section{Disclosure Statement}

The authors have no conflicts of interest.

\section{References}

1 Harris M, Howell A, Chrissohou M, Swindell RI, Hudson M, Sellwood RA: A comparison of the metastatic pattern of infiltrating lobular carcinoma and infiltrating duct carcinoma of the breast. Br J Cancer 1984;50:23-30.

-2 Lamovec J, Bracko M: Metastatic pattern of infiltrating lobular carcinoma of the breast: an autopsy study. J Surg Oncol 1991;48:28-33.

-3 Ferlicot S, Vincent-Salomon A, Medioni J, Genin P, Rosty C, Sigal-Zafrani B, et al: Wide metastatic spreading in infiltrating lobular carcinoma of the breast. Eur J Cancer 2004;40:336-341.

-4 Braman SS, Whitcomb ME: Endobronchial metastasis. Arch Intern Med 1975;135:543-547.

5 Ormerod LP, Horsfield N, Alani FS: How frequently do endobronchial secondaries occur in an unselected series? Respir Med 1998;92:599-600.

-6 Kiryu T, Hoshi H, Matsui E, Iwata H, Kokubo M, Shimokawa K, Kawaguchi S: Endotracheal/endobronchial metastases: clinicopathologic study with special reference to developmental modes. Chest 2001;119:768775.

7 Sorensen JB: Endobronchial metastases from extrapulmonary solid tumors. Acta Oncol 2004;43:73-79.

8 Matsui M, Kojima O, Kawakami S, Uehara Y, Takahashi T: The prognosis of patients with gastric cancer possessing sex hormone receptors. Surg Today 1992;22:421-425.

9 Van Velthuysen M: Expression of oestrogen receptor and loss of E-cadherin are diagnostic for gastric metastasis of breast carcinoma. Histopathology 2005;46:153-157.

10 Berx G, Cleton-Jansen AM, Strumane K, De Leeuw WJ, Nollet F, Van Roy F, et al: E-cadherin is inactivated in a majority of invasive human lobular breast cancers by truncation mutations throughout its extracellular domain. Oncogene 1996;13:1919.

-11 Cleton-Jansen AM: E-cadherin and loss of heterozygosity at chromosome 16 in breast carcinogenesis: different genetic pathways in ductal and lobular breast cancer? Breast Cancer Res 2001;4:5-8.

12 De Leeuw WJ, Berx G, Vos C B, Peterse J L, Van de Vijver MJ, Litvinov S, et al: Simultaneous loss of E-cadherin and catenins in invasive lobular breast cancer and lobular carcinoma in situ. J Pathol 1997;183:404-411. 


\section{Case Reports in Oncology}

\begin{tabular}{l|l}
\hline Case Rep Oncol 2013;6:555-560 \\
\hline DOI: $10.1159 / 000356564$ & $\begin{array}{l}\text { C 2013 S. Karger AG, Basel } \\
\text { www.karger.com/cro }\end{array}$ \\
\hline
\end{tabular}

Fernandes et al.: Gastric and Endobronchial Metastases in a Case of Lobular Breast Cancer

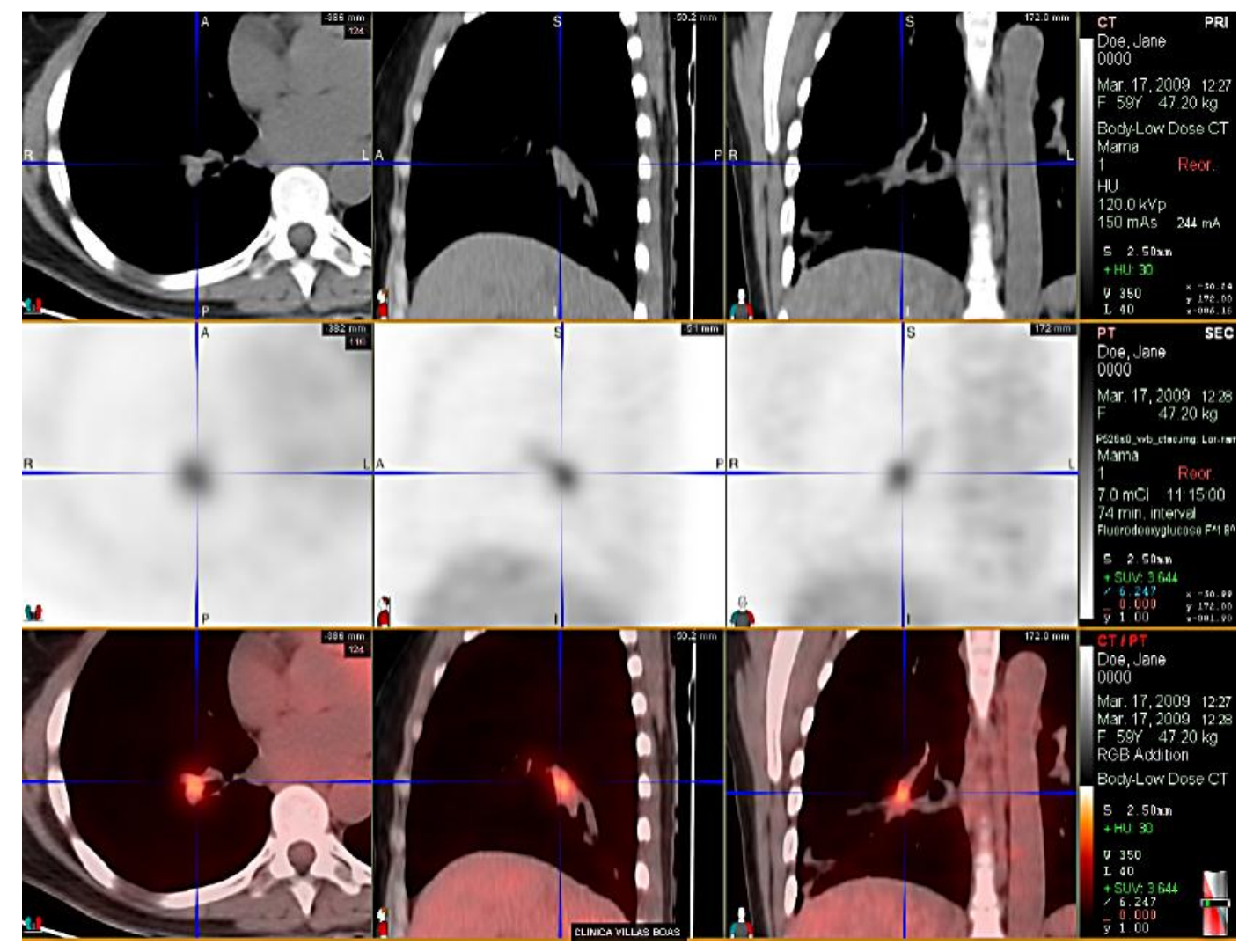

Fig. 1. ILC metastasis with airway involvement before brachytherapy. 
Fernandes et al.: Gastric and Endobronchial Metastases in a Case of Lobular Breast Cancer

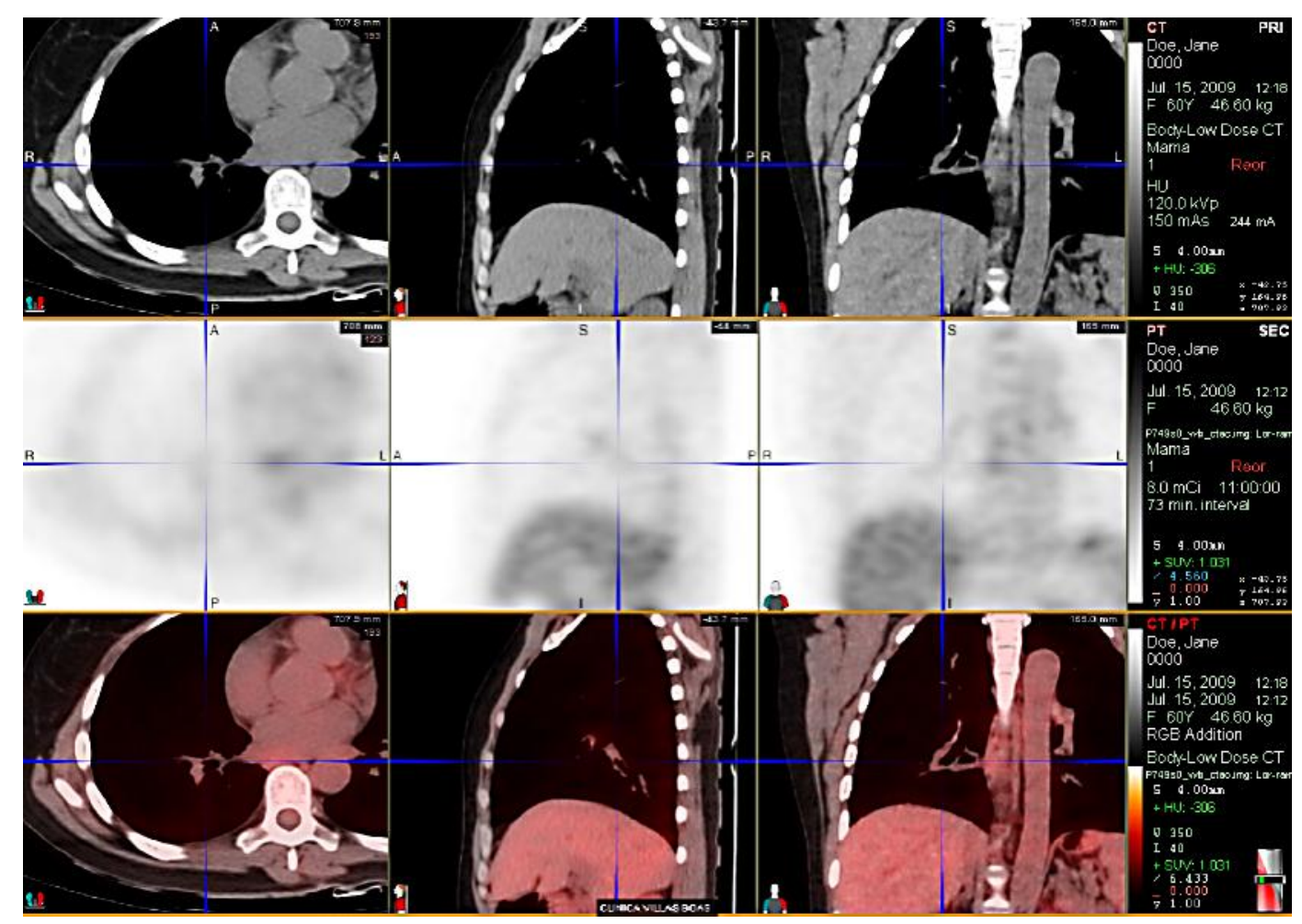

Fig. 2. ILC metastasis with airway involvement after radiotherapy.

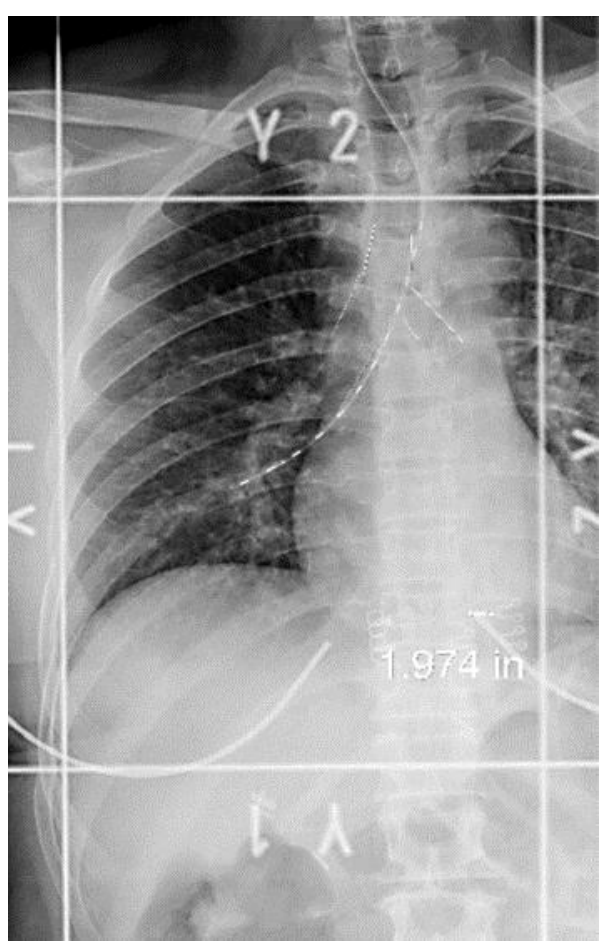

Fig. 3. Planning image for high-dose brachytherapy. 